\title{
CONTACT FORCE MODEL FOR A BEAM WITH DISCRETELY SPACED GAP SUPPORTS AND ITS APPROXIMATED SOLUTION
}

\author{
NAM-GYU PARK*, JUNG-MIN SUH, and KYEONG-LAK JEON \\ R\&D Center, KEPCO Nuclear Fuel \\ P.O. Box 14, Yuseong, Daejeon, Korea 305-353 \\ "Corresponding author. E-mail : nkpark@knfc.co.kr
}

Received November 12, 2010

Accepted for Publication April 11, 2011

This paper proposes an approximated contact force model to identify the nonlinear behavior of a fuel rod with gap supports; also, the numerical prediction of interfacial forces in the mechanical contact of fuel rods with gap supports is studied. The Newmark integration method requires the current status of the contact force, but the contact force is not given a priori. Taylor's expansion can be used to predict the unknown contact force; therefore, it should be guaranteed that the first derivative of the contact force is continuous. This work proposes a continuous and differentiable contact force model with the ability to estimate the current state of the contact force. An approximated convex and differentiable potential function for the contact force is described, and a variational formulation is also provided. A numerical example that considers the particularly stiff supports has been studied, and a fuel rod with hardening supports was also examined for a realistic simulation. An approximated proper solution can be obtained using the results, and abrupt changes from the contacting state to non-contacting state, or vice versa, can be relieved. It can also be seen that not only the external force but also the developed contact force affects the response.

KEYWORDS : Contact Force, Fuel Rod, Gap, Support

\section{INTRODUCTION}

Structures subjected to dynamic contact exist in many industrial and engineering fields[1-12]. In the nuclear industry, there are several regularly occurring examples of dynamic contacts, such as $U$ tubes in the heat exchanger and fuel rods in the reactor of nuclear power plants. In these situations, it is important to evaluate whether the structural integrity is sufficiently robust against wear. An impact model for heat exchanger tubes that considers their support width was proposed by Hassan et al., and the dynamics of the loosely supported tubes was computed $[12,13,21]$. Knudsen et al. [8] simulated the dynamics of vibro-impacts that represented fuel rods with gap supports and compared the predicted wear work rates with the experimental results.

For a fuel rod, due to the thermal relaxation of the elastic supports and the creep down of the fuel rod cladding, a gap develops between the fuel rod and the supports. Field experience has demonstrated that the gap can develop due to the relaxed support, the creep down of the fuel rod, and the growth of the grid structure after approximately one cycle of burn-up. Therefore, the dynamic impact caused by turbulent coolant flow is unavoidable. The degree of freedom in the fuel rod is restrained at a certain time when the gap closes. When the fuel rod contacts the support, a reaction force develops and this force can be regarded as an extra external force. The system is usually modeled as a beam with extra external forces or pseudo forces that simulate dynamic contact conditions [11-13]. A time domain solution can be obtained using the direct implicit integration of the governing equation using the Newmark method; however, the predicted solution may be unreliable due to unknown contact forces. Thus, a method that uses previous contact forces to estimate the contact status has been introduced [14]. Since this method uses the previous contact force as the current force, the time increment should be maintained at a small value. Another method using Taylor's expansion has also been introduced $[3,8]$. This is an approximation of the contact force using the proposed Taylor series expansion in order to improve numerical stability, but it must be noted that the slope of the contact force should be continuous at contact since Taylor's expansion is 
relevant when the first derivative is continuous.

If a structure accompanied by a contact phenomenon has a large degree of freedom, the computation time can become a serious problem. Significant model reductions can be made using a finite number of normal modes [1, 10], and computation times can be drastically reduced for a system with a large degree of freedom. However, when stiff springs are prescribed, it is recommended that the high modes are not truncated since the high frequency impact cannot be described with insufficient modes [3].

This paper proposes a continuous and differentiable contact force model that is required to calculate its tangent stiffness or Jacobian. It is also shown that the contact force model has a potential function that is convex and differentiable over the entire domain. The response is predicted using a step-by-step incremental solution. The impact of a fuel rod colliding with gap supports is solved using the same incremental approach. Numerical examples are provided to verify the reliability of the contact force model, and an application example is also listed for a more realistic situation. Fretting wear due to the grid-to-rod interaction is a root cause of fuel failure; therefore, it is required in order to accurately predict or evaluate wear on the fuel rod. The objective of this paper is to develop a primitive vibration simulator for fretting wear evaluation.

\section{STRUCTURE DESCRIPTION FOR THE MECHANICAL MODEL}

A fuel assembly is composed of approximately two hundred fuel rods supported by multiple spacer grids that consist of an arrangement of interlocked straps with springs and dimples that are formed within the straps. These springs and dimples grip and hold the fuel rods in their proper position within the fuel assembly structure. To maintain firm support conditions, the top and bottom spacer grids are usually fabricated from nickel alloy, which is less sensitive to thermal relaxation and irradiation. The mid grids, unlike the top and bottom grids, are fabricated from zirconium alloy, which is sensitive to thermal relaxation and irradiation.

Uranium pellets are loaded in the fuel rod, and there is a gap between the pellets and the inner space of the rod. The gap, despite being present, is not considered because it may be reduced due to both the creep down of the fuel rod cladding and the swelling of the pellets during service. The grid structure is complicated, and Fig. 1 shows a part of the mid grid design. In Fig. 1, a fuel rod is loaded into each cell, and a spring supports one side of each fuel rod surface while the opposite side is supported by a pair of dimples.

Fig. 2 shows the schematic of the structure used in this study. Only the unilateral contact in one plane will be considered in this study, and any out-of-plane motion will not be considered. It is assumed that the elastic supports in the top and bottom grids constantly maintain

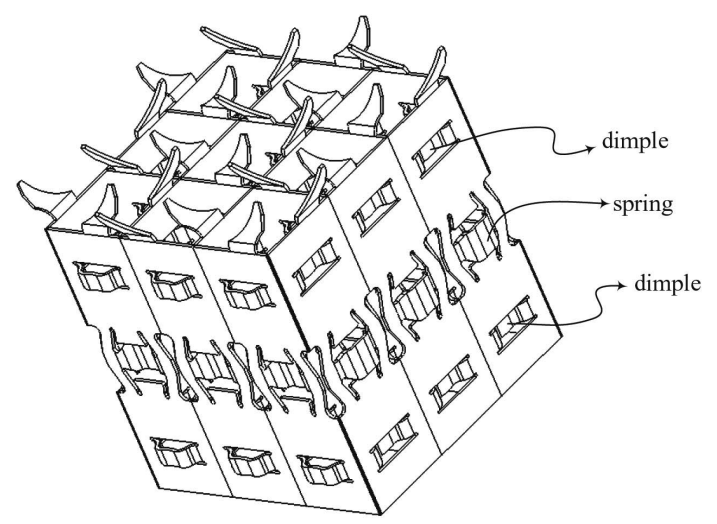

Fig. 1. Detail of Grid Assembly Structure

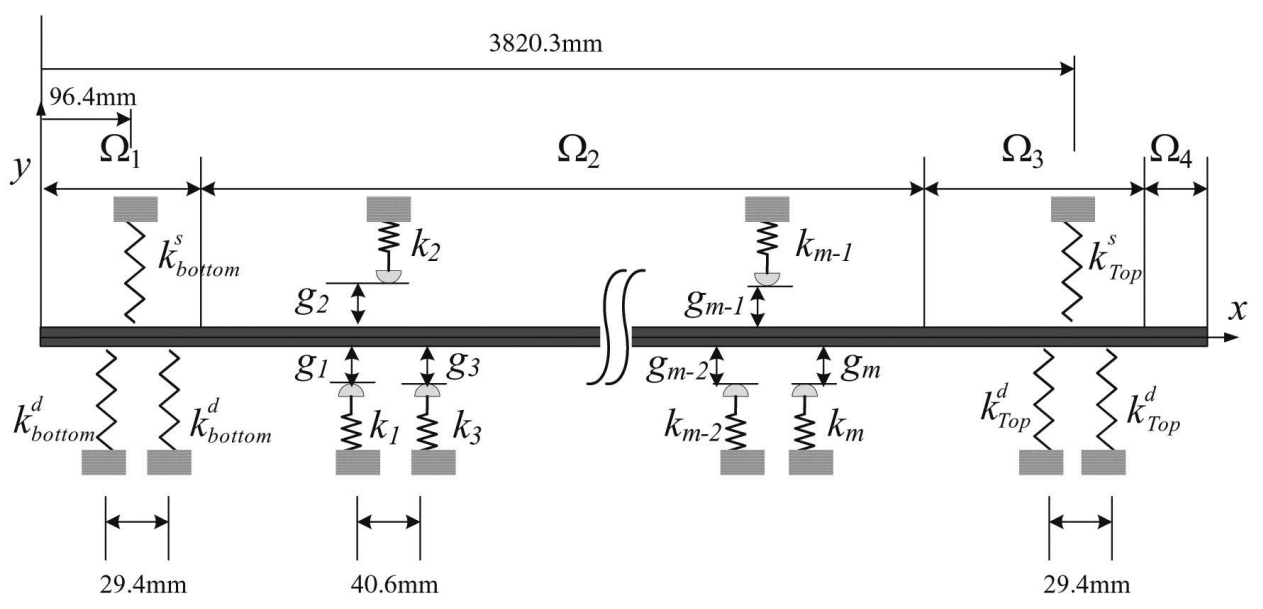

Fig. 2. Simplified Fuel Rod with Gap Supports 
contact with the fuel rod. It is also assumed that the pellet's contribution to the second moment of inertia in the structure is insignificant, and thus disregarded. Table 1 shows the mechanical properties for each domain in the structure. Since the fuel rod cross section properties such as the cross section area and density are not uniform, the structure is divided into four regions with different properties as shown in Table 1. Young's modulus is 77.95 GPa for every domain, since the fuel rod is made from zirconium alloy. Hereafter, the spring will be referred to as the upper support and the dimple will be referred to as the lower support. The upper supports in the bottom and top grids are located at $96.4 \mathrm{~mm}$ and $3820.3 \mathrm{~mm}$ from the left, respectively. The upper support in the first mid are distributed evenly over a span of $529.6 \mathrm{~mm}$. Each upper support is placed in the center of the two lower supports in the grids. The distance between the two lower supports is $40.6 \mathrm{~mm}$ for the mid grid and $29.4 \mathrm{~mm}$ for the top and bottom grids. Details of their dimensions are provided in Fig. 2.

\section{EQUATION OF MOTION AND CONTACT FORCE MODEL}

To consider the contacting force acting on the beam as an external force, the equation of motion can be written as follows $[1,3,8,10,12]$ :

$$
[M]\{\ddot{u}\}+[C]\{\dot{u}\}+[K]_{L}\{u\}=\left\{f^{e x t}\right\}+\left\{f^{C}\right\},
$$

where $\{u\}$ and $[M]$ denote the displacement vector and mass matrix, respectively. $[K]_{L},[C]$, and $\left\{f^{e x t}\right\}$ are the linear stiffness matrix, damping matrix, and external force vector, respectively. For the linear supports, the last term in Eq. (1), $\left\{f^{c}\right\}$, is the contact force. The $i^{\text {th }}$ component can be written as:

$$
f_{i}^{C}=\left\{\begin{array}{cc}
-k_{i}^{C}\left(u_{i}-g_{i}\right) & \text {, if } u_{i}>g_{i} \\
0 & \text {, if } u_{i} \leq g_{i}
\end{array},\right.
$$

where $g_{i}$ and $k_{i}^{c}$ denote the $i^{\text {th }}$ gap distance and $i^{\text {th }}$ support spring constant, respectively. Note that Eq. (2) is effective for the upper supports only: the supports above the beam in Fig. 2. For the lower supports, the contact force is active only when the displacement is lower than the gap. From this point, only the upper support will be discussed.

Eq. (1) must be satisfied at all times and assuming the solution at time $t$ is known, the displacement and its time derivatives at time, $t+\Delta t$, are expressed as presented in Eq. (3) for the Newmark method:

$$
\begin{aligned}
& \{u\}_{t+\Delta t}=\{u\}_{t}+\Delta t\{\dot{u}\}_{t}+\frac{1}{2} \Delta t^{2}(1-2 \beta)\{\ddot{u}\}_{t}+\beta \Delta t^{2}\{\ddot{u}\}_{t+\Delta t} \\
& \{\dot{u}\}_{t+\Delta t}=\{\dot{u}\}_{t}+(1-\gamma) \Delta t\{\ddot{u}\}_{t}+\gamma \Delta t\{\ddot{u}\}_{t+\Delta t} \\
& \{\ddot{u}\}_{t+\Delta t}=\frac{1}{\beta \Delta t^{2}}\left(\{u\}_{t+\Delta t}-\{u\}_{t}\right)-\frac{1}{\beta \Delta t}\{\dot{u}\}_{t}-\frac{1-2 \beta}{2 \beta}\{\ddot{u}\}_{t},
\end{aligned}
$$

where $\Delta t$ is the time increment. Usually, considering the stability of the integration [22], $1 / 4$ and $1 / 2$ are preferred for $\beta$ and $\gamma$, respectively. When substituting the velocity and the acceleration of Eq. (3) into Eq. (1), the solution for the displacement at time $t+\Delta t$ is expressed as:

$$
[K]\{u\}_{t+\Delta t}=\left(\left\{f^{e x t}\right\}_{t+\Delta t}+\left\{f^{C}\right\}_{t+\Delta t}+\{F\}\right)
$$

where $[\boldsymbol{K}]$ is dynamic stiffness, which is defined as:

$$
[K]=[K]_{L}+\frac{1}{\beta \Delta t^{2}}[M]+\frac{\gamma}{\beta \Delta t}[C],
$$

where $\{F\}$ in Eq. (4) is another force vector that results from the inertia and damping at time $t$, and it can be defined as in Eq. (6):

$$
\begin{aligned}
\{F\}=[M] & \left(\frac{1}{\beta \Delta t^{2}}\{u\}_{t}+\frac{1}{\beta \Delta t}\{\dot{u}\}_{t}+\frac{1-2 \beta}{2 \beta}\{\ddot{u}\}_{t}\right) \\
& +[C]\left(\frac{\gamma}{\beta \Delta t}\{u\}_{t}+\frac{\gamma-\beta}{\beta}\{\dot{u}\}_{t}+\Delta t \frac{2 \beta-\gamma}{2 \beta}\{\ddot{u}\}_{t}\right) .
\end{aligned}
$$

The solution can be found based on Eq. (4), but it should be noted that the contact force, $\left\{f^{C}\right\}$, is unknown at time $t+\Delta t$. Johansson [3] attempted to solve this problem using Taylor's expansion of the contact force. That is:

$$
\left\{f^{C}\left(u_{t+\Delta t}\right)\right\}=\left\{f^{C}\left(u_{t}\right)\right\}+\frac{\partial\left\{f^{C}\right\}}{\partial\{u\}}\left\{u_{t+\Delta t}-u_{t}\right\} .
$$

Table 1. Material and Sectional Properties of the Fuel Rod

\begin{tabular}{c|c|c|c}
\hline Domain & density $\left(\mathrm{kg} / \mathrm{m}^{3}\right)$ & cross section area $\left(\mathrm{m}^{2}\right)$ & area $2 \mathrm{nd} \mathrm{moment} \mathrm{of} \mathrm{inertia}\left(\mathrm{m}^{4}\right)$ \\
\hline$\Omega_{1}$ & $5.253 \mathrm{E} 4$ & $6.581 \mathrm{E}-5$ & $3.432 \mathrm{E}-10$ \\
\hline$\Omega_{2}$ & $4.186 \mathrm{E} 5$ & $1.548 \mathrm{E}-5$ & $1.420 \mathrm{E}-10$ \\
\hline$\Omega_{3}$ & $9.893 \mathrm{E} 4$ & $1.548 \mathrm{E}-5$ & $3.420 \mathrm{E}-10$ \\
\hline$\Omega_{4}$ & $4.107 \mathrm{E} 4$ & $6.581 \mathrm{E}-5$ & $32 \mathrm{E}-10$ \\
\hline
\end{tabular}


This expression is meaningful when the contact force is differentiable, but the contact force model in Eq. (2) is not differentiable at $u_{i}=g_{i}$. The solid line in Fig. 3 shows the graphical representation of the model, and it is continuous; however, the slope is not visibly continuous.

To relieve this drawback, an approximated contact force can be defined as:

$$
f_{i}^{C}=\left\{\begin{array}{ccc}
-k_{i}^{C}\left(u_{i}-g_{i}\right) & , & \text { if } u_{i}>g_{i}+\varepsilon \\
-\frac{k_{i}^{C}}{4 \varepsilon}\left(u_{i}-\left(g_{i}-\varepsilon\right)\right)^{2} & , & \text { if } g_{i}-\varepsilon \leq u_{i} \leq g_{i}+\varepsilon \\
0 & , & \text { if } u_{i}<g_{i}-\varepsilon
\end{array}\right.
$$

where $\varepsilon$ denotes a reasonably small positive number. Essentially, Eq. (8) denotes the continuously varying contact force shown in Fig. 3. Clearly, the contact force is not only continuous, but the slope is also continuous over the entire displacement domain. With Eq. (8), substituting Eq. (7) to Eq. (4), it leads to:

$$
([K]-[T])\{u\}_{t+\Delta t}=\left(\left\{f^{e x t}\right\}_{t+\Delta t}+\left\{f^{C}\right\}_{t}-[T]\{u\}_{t}+\{F\}\right),
$$

where $[T]$ is the tangential stiffness of the contact force, $\frac{\partial\left\{f^{C}\right\}}{\partial\{u\}}$. Considering that $[K]$ is linear and constant while $[T]$ is nonlinear, Eq. (9) can be rewritten as:

$$
\{u\}_{t+\Delta t}=[K]^{-1}\left(\{P\}_{t+\Delta t}+[T]\{u\}_{t+\Delta t}\right),
$$

where $\{P\}$ indicates the terms in the right side of Eq. (9). An approximated solution, especially for a nonlinear structure, can be found using the Picard iteration [15]. Furthermore, an iterative solution based on Eq. (10) is:

$$
\text { (r) }\{u\}_{t+\Delta t}=[K]^{-1}\left(\{P\}_{t+\Delta t}+^{(r-1)}[T]^{(r-1)}\{u\}_{t+\Delta t}\right),
$$

where the left superscript denotes the iteration number.

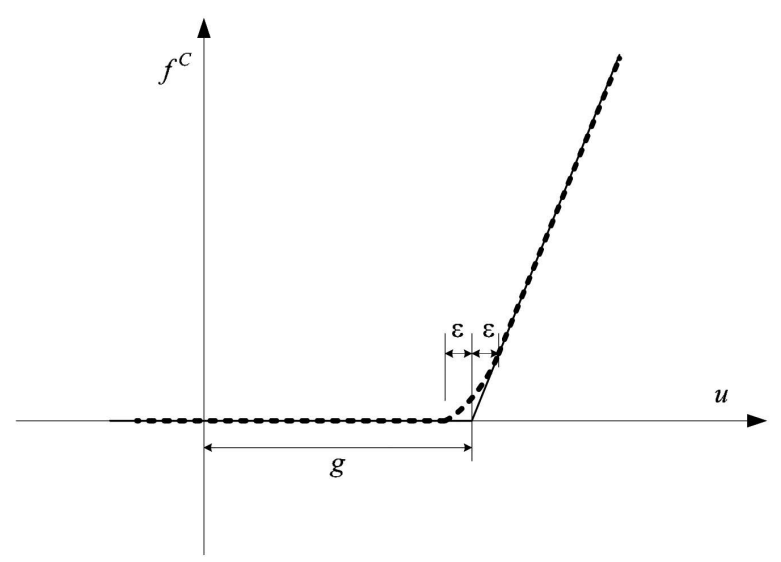

Fig. 3. Approximated Contact Force (Dotted Line)
The initial values for the iterative terms are:

$$
\begin{aligned}
& { }^{(0)}[T]=\left[T\left(u_{t}\right)\right] \\
& { }^{(0)}\{u\}_{t+\Delta t}=\{u\}_{t}
\end{aligned} \text {. }
$$

Since the solution is iteratively obtained, convergence conditions are required to verify the integrity of the solution. Usually, a norm for the residual force or a norm of the incremental solution is used for these conditions. The convergence of the problem is dependent on the time increment, and the theory for the convergence of the integration method has already been discussed and well established by Hughes [22].

\section{POTENTIAL FUNCTION OF THE CONTACT FORCE AND VARIATIONAL FORMULATION}

The dynamic equation for the fuel rod structure can be restated using the variational method perspective: the system can be thought of as a beam with resilient obstacles. It is well known that the dynamic contact problem can be characterized using a variational inequality $[16,17]$. The variational formulation is required to demonstrate the uniqueness and existence of the solution. As will be shown in this section, because the suggested contact force model is continuous and convex, it can be stated that the solution is unique according to the Lax-Milgram lemma [23]. For a specified time duration, $\tau$, the first variation of the total potential energy of the structure satisfies the inequality rather than the equality [15].

$$
\begin{array}{r}
\delta \Pi(u)=\int_{0}^{\tau}\left[\int_{\Omega} \rho\left(\frac{\partial u}{\partial t}\right)\left(\frac{\partial \delta u}{\partial t}\right) d \Omega-\int_{\Omega} E I\left(\frac{\partial^{2} u}{\partial x^{2}}\right)\left(\frac{\partial^{2} \delta u}{\partial x^{2}}\right) d \Omega\right. \\
\left.-\delta \Phi+\int_{\Omega} f(x, t) \delta u d \Omega\right] d t \geq 0,
\end{array}
$$

where $E, I, \rho$, and $f$ are Young's modulus, the second area moment, the density of the fuel rod, and the given external force, respectively. $\Omega$ denotes the entire domain for the structure, and $\delta$ denotes the first variation of the total potential energy. It should be noted that the displacement, $u$, is a kinematically admissible displacement field that satisfies the essential boundary condition. $\Phi$ is the potential energy of the supports in the top and bottom grids. Thus, $\Phi$ is a summation of each potential of the supports in the top and bottom grids, and each one is expressed as:

$$
\Phi_{i}=\frac{1}{2} k_{i} u_{i}^{2},
$$

where $u_{i}$ is the motion at the support location.

It has also been theoretically proven that the variational inequality becomes equality when the convex and Gateaux differentiable potential function resulting from contact can be defined [16]. When the potential function of the 
contact force, which is convex and differentiable, is found, then the total potential can be written as:

$$
\begin{array}{r}
\Pi=\int_{0}^{\tau} \int_{\Omega} \frac{1}{2} \rho\left(\frac{\partial u}{\partial t}\right)^{2} d \Omega d t-\int_{0}^{\tau} \int_{\Omega} \frac{1}{2} E I\left(\frac{\partial^{2} u}{\partial x^{2}}\right)^{2} d \Omega d t \\
-\Phi+\int_{0}^{\tau} \int_{\Omega} f(x, t) u d \Omega d t-\int_{0}^{\tau} \sum_{i=1}^{m} \Psi_{i} d t
\end{array}
$$

where the last term is from the contacting force in the mid grid supports and $m$ is the number of contact points. An approximated potential function for the suggested contact force model can be defined as:

$$
\Psi_{i}^{\varepsilon}=\left\{\begin{array}{ccc}
\frac{1}{2} k_{i}\left(u_{i}-g_{i}\right)^{2}+\frac{1}{6} k_{i} \varepsilon^{2} & , & \text { if } u_{i}>g_{i}+\varepsilon \\
\frac{k_{i}}{12 \varepsilon}\left(u_{i}-\left(g_{i}-\varepsilon\right)\right)^{3} & , & \text { if } g_{i}-\varepsilon \leq u_{i} \leq g_{i}+\varepsilon . \\
0 & , & \text { if } u_{i}<g_{i}-\varepsilon
\end{array}\right.
$$

It is clear that Eq. (16) is continuous and convex as shown in Fig. 4. It is also differentiable at the two boundary points, $g_{i}-\varepsilon$ and $g_{i}+\varepsilon$, and its gradient denotes the contact force, Eq. (8). Therefore, the variational equality problem can be defined, and an approximated solution can be obtained. The constant terms in Eq. (16) are arbitrary. Another potential function can be a candidate when the continuity is satisfied. This work prefers zero potential at the non-contact condition, $u_{i}<g_{i}-\varepsilon$, since it is physically more meaningful because the support is not effective before the gap closes.

Using Eq. (16), the variation of Eq. (15) equals to zero, and it can be written as in Eq. (17):

$$
\begin{aligned}
& \delta \Pi_{\varepsilon}=-\int_{0}^{\tau} \int_{\Omega} \rho \frac{\partial^{2} u}{\partial t^{2}} \delta u d \Omega d t-\int_{0}^{\tau} \int_{\Omega} E I\left(\frac{\partial^{2} u}{\partial x^{2}}\right)\left(\frac{\partial^{2} \delta u}{\partial x^{2}}\right) d \Omega d t \\
& -\delta \Phi+\int_{0}^{\tau} \int_{\Omega} f(x, t) \delta u d \Omega d t \\
& -\int_{0}^{\tau} \sum_{i=1}^{m} \delta \Psi_{i}^{\varepsilon} d t=0
\end{aligned}
$$

As $\varepsilon$ approaches 0 , a more exact solution can be found, and the finite element formulation of Eq. (17) is equal to Eq. (1) using the contact force in Eq. (8).

\section{NUMERICAL ANALYSIS}

A finite element model was prepared based on the mechanical properties presented in Table 1 and the configuration described in the previous section. The model consists of 62 nodes, and the supports in the top and bottom grids were always in contact with the beam. It is assumed that the supports in the top and bottom grids behave linearly, and their spring constants are listed in Table 2. The contact stiffness in Table 2 is based on the measured static load-deflection characteristics. Eighteen (18) contact points, which represent the six mid grids, were distributed along the beam.

\subsection{Case Study: Harmonic Excitation}

When the contact stiffness is very large, the contacting nodes of the beam cannot move above or below the gaps. In this example, the stiffness of the 18 supports in the mid grids is set to $5.0 \mathrm{E} 5 \mathrm{~N} / \mathrm{m}$, which is approximately eight times larger than that of the upper support in the real structure. Also, all 18 gaps are set to $0.25 \mathrm{~mm}$. Two external sinusoidal loads are applied to randomly chosen points that were placed at $223 \mathrm{~mm}$ and $2052 \mathrm{~mm}$ from the origin. The magnitude of the loads was $8.9 \mathrm{~N}$ and the excitation frequency was $10 \mathrm{~Hz}$ for both loads. Rayleigh damping is used, and the damping for the simulation is $[C]=\alpha[K]$. The damping parameter, $\alpha$, is selected to damp

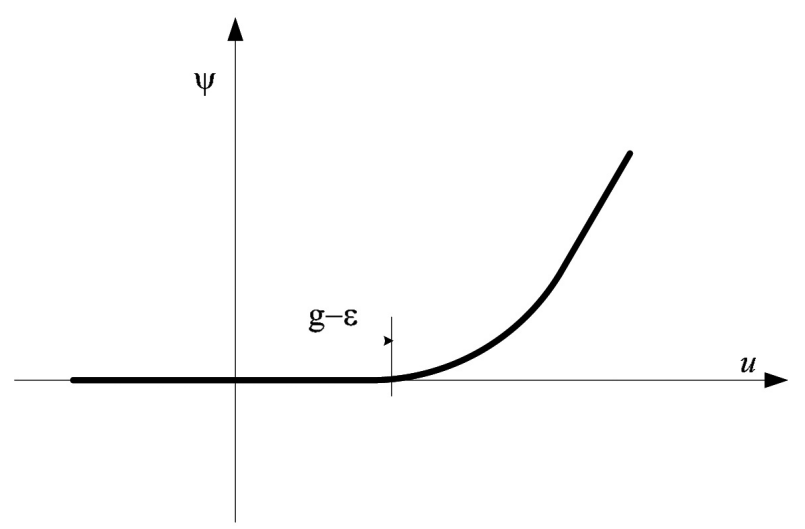

Fig. 4. Potential Energy of the Approximated Contact Force

Table 2. Spring Constants for the Top and the Bottom Grids

\begin{tabular}{c|c|c}
\hline & Upper support (N/m) & Lower support (N/m) \\
\hline Top grid & $1.51 \mathrm{E} 5$ & $2.89 \mathrm{E} 5$ \\
\hline Bottom grid & $5.41 \mathrm{E} 4$ & $1.59 \mathrm{E} 5$ \\
\hline
\end{tabular}


out all high frequency components after the $50^{\text {th }}$ natural frequency. Therefore, according to the basic modal analysis theory, the damping coefficient, $\varsigma_{n}$, can be expressed as:

$$
\varsigma_{n}=\pi \alpha f_{n},
$$

where $f_{n}$ denotes the $n^{\text {th }}$ natural frequency. Considering that the $50^{\text {th }}$ natural frequency of the structure is $1.188 \mathrm{E} 3 \mathrm{~Hz}$ and assuming that the $50^{\text {th }}$ mode is critically damped $(\varsigma=1)$, it is found that $\alpha$ should be greater than $2.68 \mathrm{E}-4$. In this case, $2.7 \mathrm{E}-4$ was used for $\alpha$. The time increment, $\Delta t$, in the example is $2.0 \mathrm{E}-5 \mathrm{sec}$. According to Au-Yang's work [19], the effective random pressure spectrum is within approximately $100 \mathrm{~Hz}$ when an estimated cross flow velocity of $1 \mathrm{~m} / \mathrm{sec}$ in the fuel bundle is used. Therefore, it is thought that the simulation should be sufficiently conservative because the external pressure spectrum range is within the considered number of modes.

Figure 5 shows the global deflection shape at the specified instances when $\varepsilon$ is 0.0025 . The two peaks at $0.01 \mathrm{sec}$ are due to the applied external loads at those positions. The restrained motions at the gap supports are also shown in Fig. 6(a) and Fig. 7(a). The motion and contact force for Fig. 6 and Fig. 7 are derived from the contacting nodes in the third mid grid, and the results include the solution at $\varepsilon=0$. It is apparent that Eq. (8) with $\varepsilon=0$ is reduced to Eq. (2) and that, in this case, the first derivative of the contact force is not continuous at the contact condition. Considering this, the contact force, based on Eq. (8), must be infinitive when $\varepsilon=0$, but it is not expected that proper solutions are able to be obtained in those conditions. As is shown in detail B of Fig. 8(a), this means that there is no instance where the displacement at the contacting node coincides precisely with the gap distance. If the current motion coincides with the gap distance, the first derivative of the contact force cannot

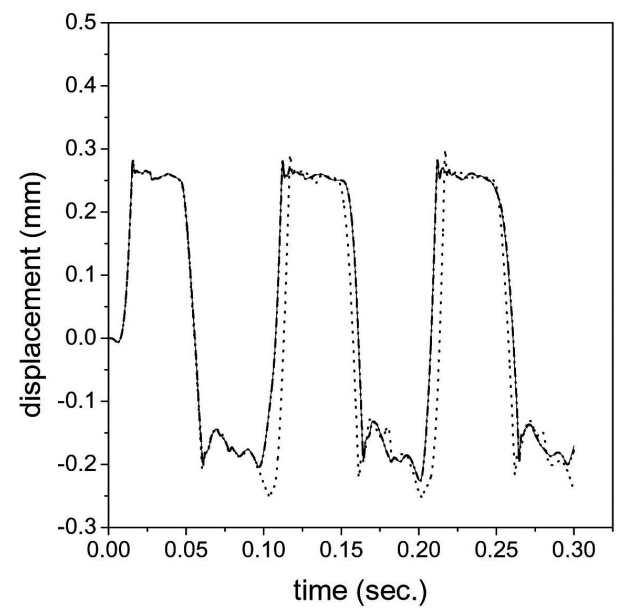

(a) displacement be defined and a proper solution cannot be obtained. Considering the given gap distance, the results in Fig. 6(a) and Fig. 7(a) are reasonable because the motions are restricted to approximately $0.25 \mathrm{~mm}$ and $-0.25 \mathrm{~mm}$, respectively. Figure 6(b) and Fig. 7(b) show the contact forces, and a negative contact force develops in the upper support while a positive one can be seen in the lower support. The downward motion for the contacting nodes corresponding to the upper supports is also restricted by the two lower supports as shown in Fig. 2. Therefore, the motion in the contacting nodes for the upper supports is more restrictive than that of the nodes in the lower supports. As $\varepsilon$ approaches zero, the variation of the results becomes indistinguishable. As shown in Fig. 6 and Fig. 7, the results in the case of $\varepsilon=0$ are indiscernible from those when $\varepsilon=2.5 E-4$.

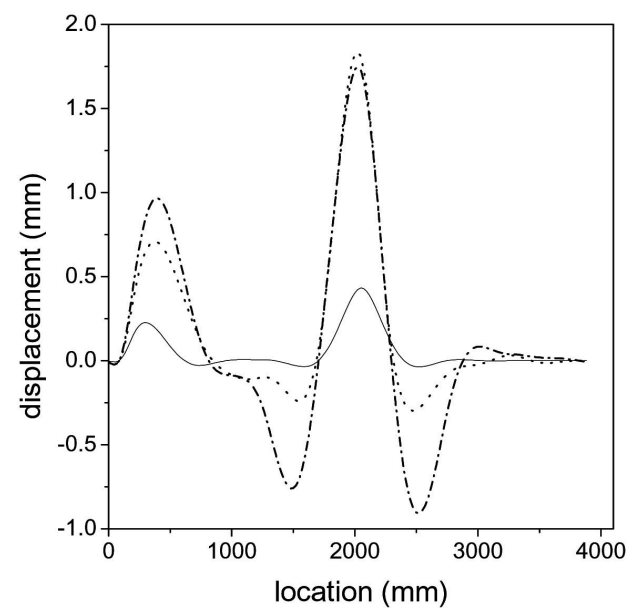

Fig. 5. Deflected Shapes (Solid : $0.01 \mathrm{sec}$., Dot : $0.02 \mathrm{sec}$, Dash Dot : $0.03 \mathrm{sec}$.

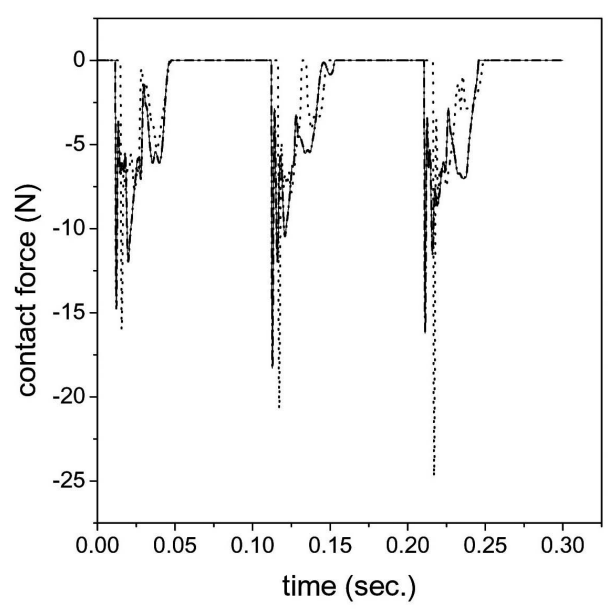

(b) contact force

Fig. 6. Displacement and Contact Force at the Upper Support (Dot : $\varepsilon=2.5 E-3$, Dash Dot : $\varepsilon=2.5 E-4$, Solid : $\varepsilon=0$ ) 
The force-displacement plots at the same contacting point are delineated in Fig. 8 as a function of $\varepsilon$, The macroscopic views show no difference in each case.
Multiple force-deflection curves can be seen in the magnified view of Fig. 8(a), which indicates that the noncontacting state jumps to the contacting state or vice versa.
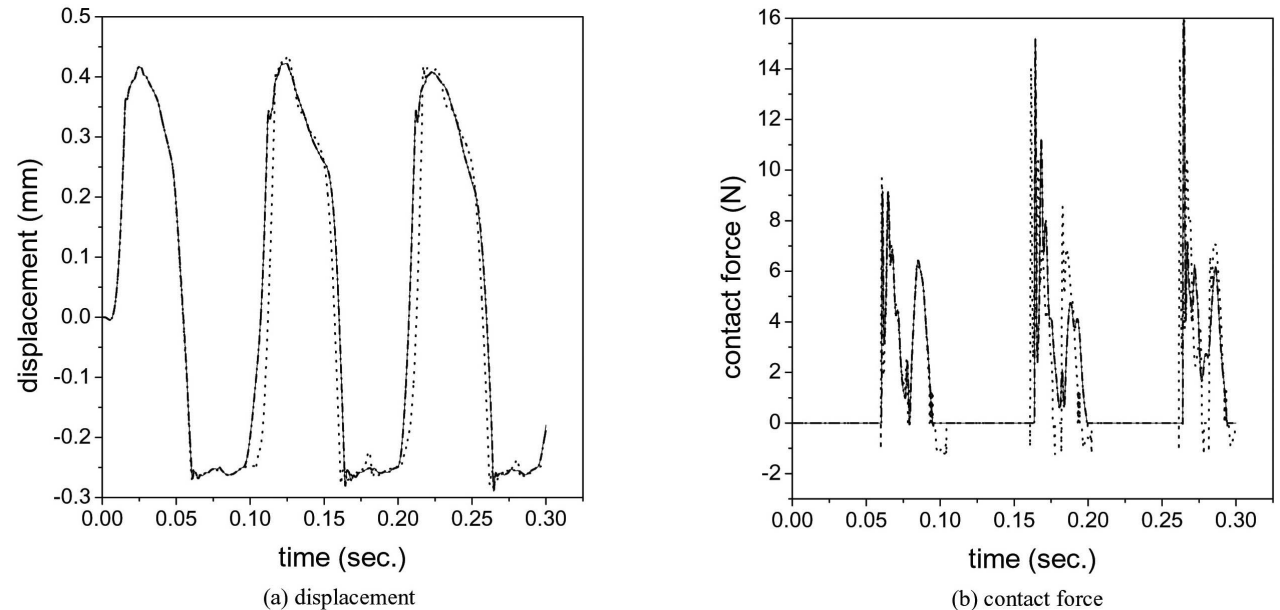

Fig. 7. Displacement and Contact Force at the Lower Support (Dot : $\varepsilon=2.5 E-3$, Dash Dot : $\varepsilon=2.5 E-4$, Solid : $\varepsilon=0$ )

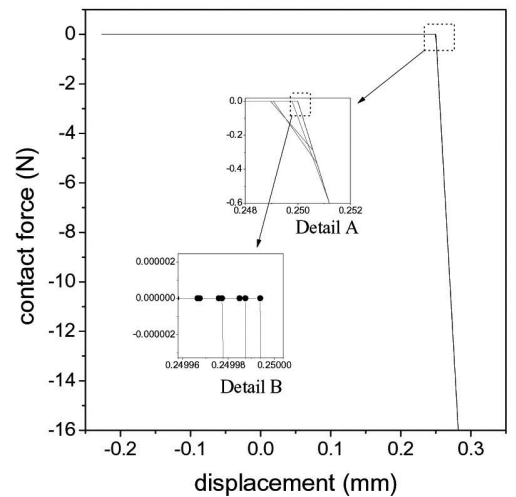

(a) $\varepsilon=0 \quad(\Delta t=2.0 E-5 \mathrm{sec}$. $)$

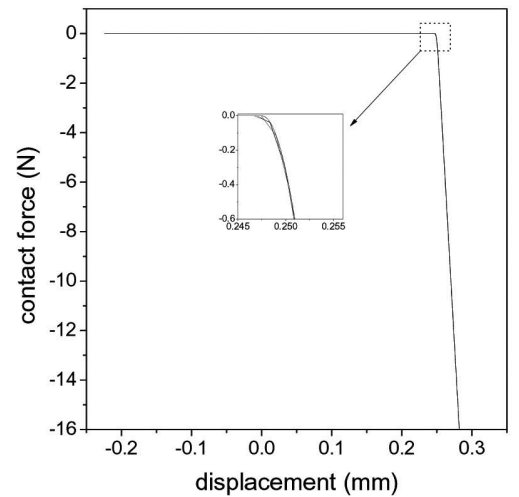

(c) $\varepsilon=2.5 e-3(\Delta t=2.0 E-5 \mathrm{sec}$. $)$

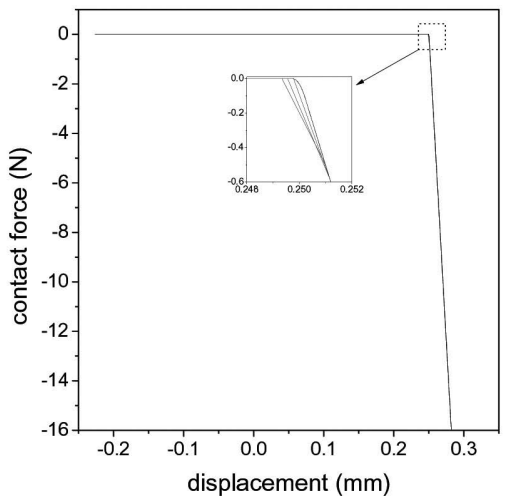

(b) $\varepsilon=2.5 e-4(\Delta t=2.0 E-5 \mathrm{sec}$. $)$

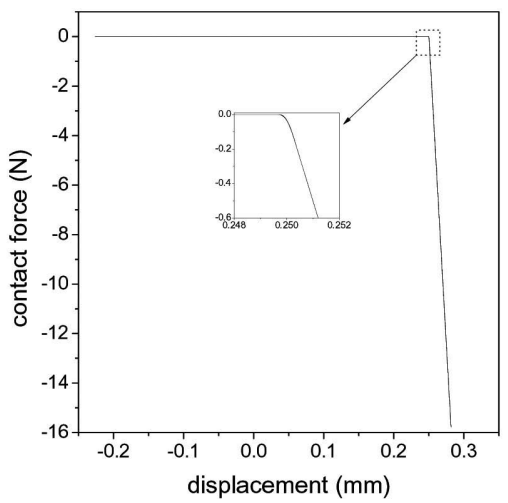

(d) $\varepsilon=2.5 e-4(\Delta t=2.0 E-6 \mathrm{sec}$. $)$

Fig. 8. Force-displacement Curves Including Magnified Views 
This is because the defined contact model, Eq. (2) or Eq. (8) where $\varepsilon=0$, is not smooth at the contacting point. As mentioned above, although the displacement at the contacting node never coincides exactly with the gap distance, it must be noted that the contact force at the next time step cannot be defined if the current displacement coincides with the gap distance. Multiple curves can also be seen for $\varepsilon=2.5 E-4$ in Fig. 8(b), but the multiple curves lie more closely together than those of Fig. 8(a). The contact model with a small positive $\varepsilon$ in the specified region behaves as a buffer spring that prevents any abrupt changes of the contacting force. Therefore, more stable and smoother results are expected, but the accuracy will be reduced as $\varepsilon$ becomes larger, as shown in Figs. 8(a) to $8(\mathrm{c})$. However, more reliable results can be obtained with a much smaller time increment, as shown in Fig. 8(d). Fig. 9 represents a power spectrum of the motion, and it is shown that the super-harmonic and sub-harmonic components of the excitation frequency, $10 \mathrm{~Hz}$, exist due to the developed contacting force.

\subsection{Case Study: Application Example}

Particularly for a nuclear fuel grid structure, hardening supports are more realistic than linear ones; for example, Fig. 10 shows the load-deflection test result of a support in a mid grid structure. It is derived from the four cycles of the loading and unloading conditions, and it can be seen that the contact force can be approximated using a polynomial function. The load-deflection curve shows that the support hardens as the displacement grows. These types of hardening effects can be simulated using a restoring force that is proportional to the cubic displacement, as with the Duffing equation [18]. In this example, thus, the hardening support can be expressed as in Eq. (19):

$$
f_{i}^{C}=\left\{\begin{array}{cl}
-k_{i}^{C}\left(u_{i}-g_{i}\right)^{3} & , \text { if } u_{i}>g_{i} \\
0 & \text {, if } u_{i} \leq g_{i}
\end{array}\right.
$$

Based on Eq. (19), it is true that both the contact force and its slope are continuous at $u_{i}=g_{i}$. Therefore, the buffer regions as in Eq. (8) are not required. Under these conditions, the individual potential energy of the gap support can be expressed as:

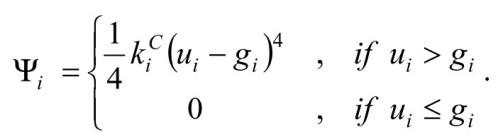

It is true that the potential function is convex and differentiable; thus, a unique solution can be found. The spring constants that best fit the measured data were chosen for the hardened supports: $2.48 \mathrm{E} 5 \mathrm{~N} / \mathrm{m}$ and $6.73 \mathrm{E} 4 \mathrm{~N} / \mathrm{m}$ for the lower support and the upper support, respectively. It was reported that the gap sizes in the pressurized water reactor (PWR) fuel assembly after the

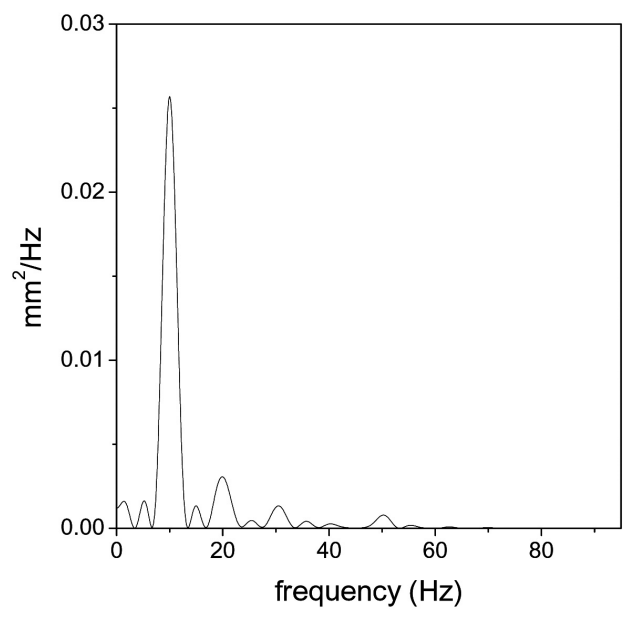

Fig. 9. Power Spectral Density Function in the Response

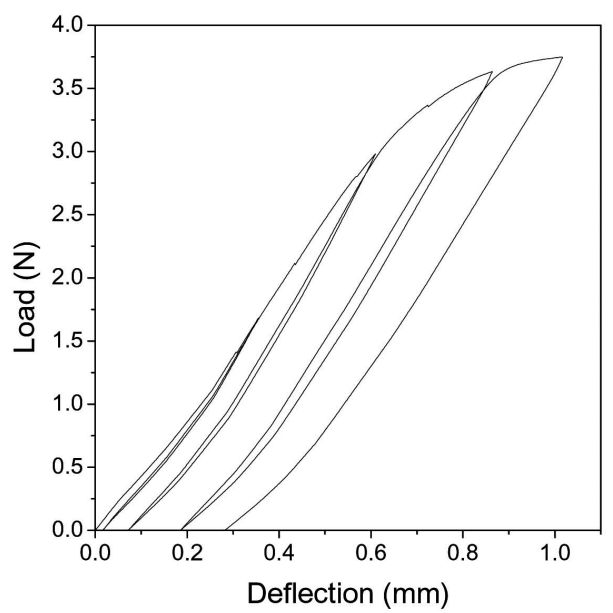

Fig. 10. Typical Load-deflection Curves for a Support

finishing service were varied, but the largest was approximately $0.05 \mathrm{~mm}$; thus, the gap distance in this example was $0.05 \mathrm{~mm}$ for every gap support.

The fuel rod is exposed to a turbulent flow, thus the bandwidth limited white noise will be applied to every middle node in each span. Field experience has confirmed that the average cross flow velocity between the two adjacent fuels is approximately $1 \mathrm{~m} / \mathrm{sec}$. According to the normalized random power spectral density [19], when a $9.1 \mathrm{~mm}$ diameter rod is used, the major frequency components are distributed below $100 \mathrm{~Hz}$. Although the experimental condition is unrealistic, the experiment results demonstrate that the lift force and drag force power spectrum was less than $1 \mathrm{~N}^{2} / \mathrm{Hz}$ [20]. Therefore, it is assumed that their frequency components are distributed 


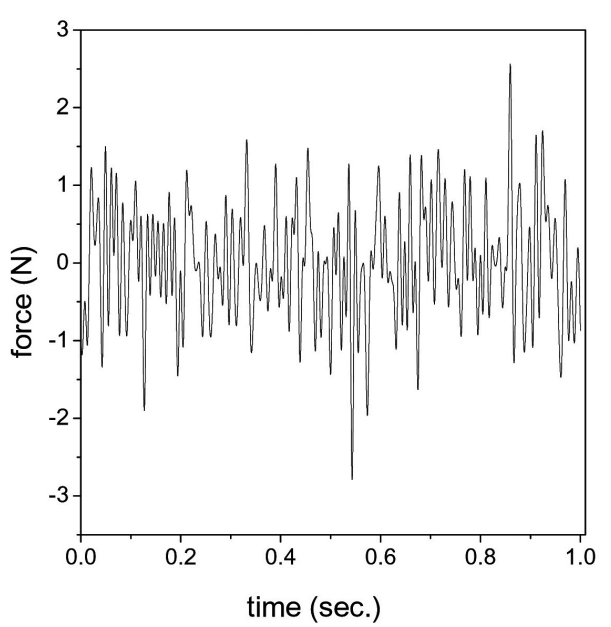

(a) external force in time domain

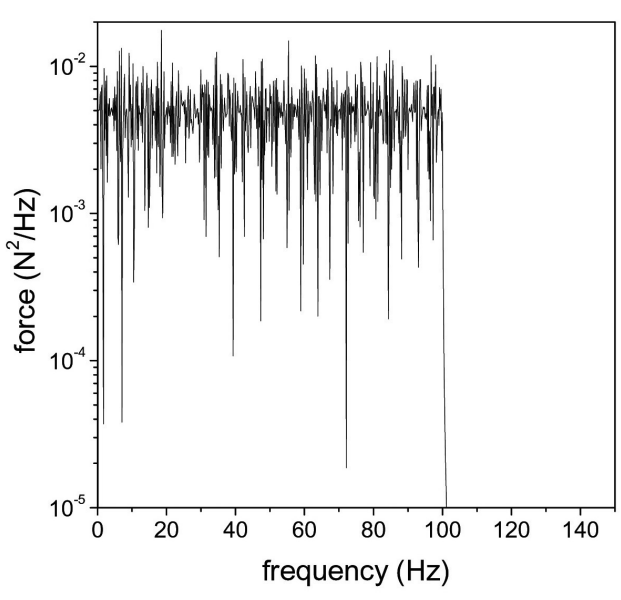

(b) power spectral density of the external force (negative frequency components are not shown)

Fig. 11. Applied Random force at the Middle of the First Span from the Left

between 0 and $100 \mathrm{~Hz}$, and that the power spectral density function is defined to provide $1 \mathrm{~N}$ in the root mean square (RMS) value. Fig. 11 shows an example of the external force in the time and frequency domains.

The Newton-Raphson method [15], which requires the Jacobian of the stiffness matrix, was chosen to solve the equation, since the contact force is sufficiently smooth. The residual force of Eq. (9) at time $t+\Delta t$ can be defined as:

$$
\begin{aligned}
\{R(u+\Delta u)\}= & ([K]-[T])\{u\}_{t+\Delta t} \\
& -\left(\left\{f^{e x t}\right\}_{t+\Delta t}+\left\{f^{C}\right\}_{t}-[T]\{u\}_{t}+\{F\}\right) .
\end{aligned}
$$

Expanding Eq. (2) in a Taylor series, neglecting the higher order terms in $\{u\}$ and noting that all external forces are fixed, the incremental solution can be obtained as follows:

$$
\{\Delta u\}_{t+\Delta t}=\left[K_{T}(u)\right]^{-1}\left(\{\hat{f}\}_{t+\Delta t}-[K(u)-T(u)]_{t}\{u\}_{t}\right),
$$

where $\{\hat{f}\}$ denotes $\left\{f^{e x t}\right\}_{t+\Delta t}+\left\{f^{c}\right\}_{t}-[T]\{u\}_{t}+\{F\}$. The tangential stiffness, $\left[\boldsymbol{K}_{T}\right]$ in Eq. (22), can be written as:

$$
\left[K_{T}\right]=\frac{\partial\{R\}}{\partial\{u\}}=[K]-[T]-\sum_{j=1}^{m} \frac{\partial^{2} f^{C}\left(u_{j}\right)}{\partial u_{j}^{2}} u_{j}\{b\}_{j}\{b\}_{j}^{T r},
$$

where $\{b\}_{j}$ is composed of one at the $j^{\text {th }}$ support position and zeros elsewhere, and the superscript, $T r$, denotes a transpose. The increment can also be obtained iteratively, and the solution at the next time step can be written as:

$$
\{u\}_{t+\Delta t}=\{u\}_{t}+\{\Delta u\}_{t+\Delta t} .
$$

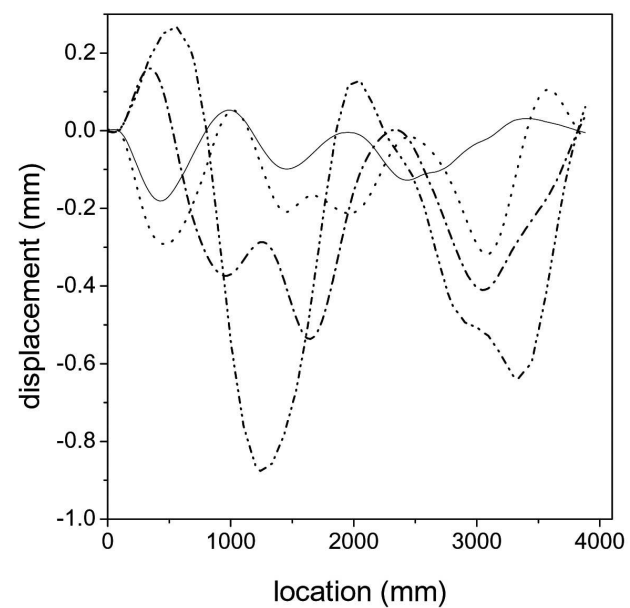

Fig. 12. Deflected Shapes (Solid : $0.01 \mathrm{sec}$, Dot : $0.02 \mathrm{sec}$., Dash Dot : $0.03 \mathrm{sec}$., Dash Dot Dot : $0.04 \mathrm{sec}$.)

It should be noted that because the contact force is estimated based on Taylor's expansion, the NewtonRaphson method requires a second derivative of the contact force as shown in Eq. (23).

The time increment, $\Delta t$, in the application example is also $2.0 \mathrm{E}-5 \mathrm{~s}$, and the same damping from the previous example is used. The deflected shapes are delineated in Fig. 12 in a few seconds, and in the time history of the contacting node on the beam in Fig. 13. Since the applied forces are band limited, the responses are also band limited as shown in Fig. 13. The dominant frequency components in the response signals are distributed within $50 \mathrm{~Hz}$. This 


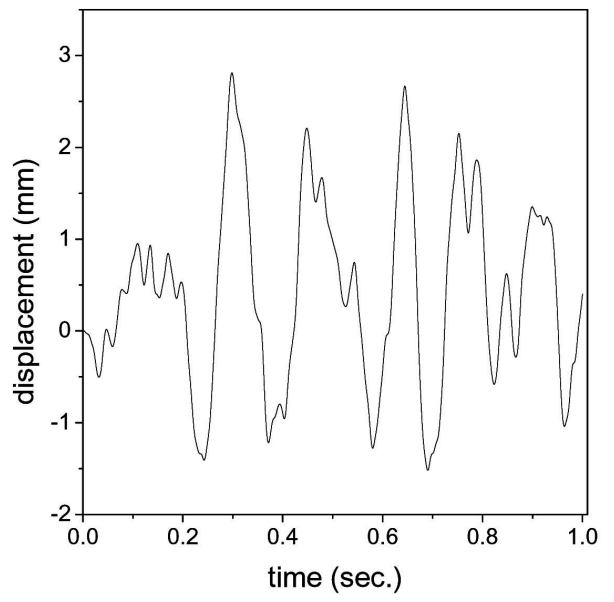

(a) displacement in time domain

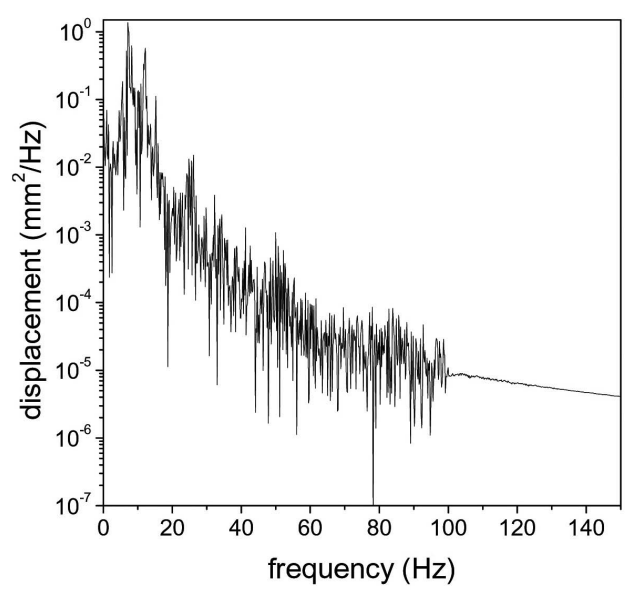

(b) displacement power spectral density

Fig. 13. Response Signal in Time and Frequency Domain

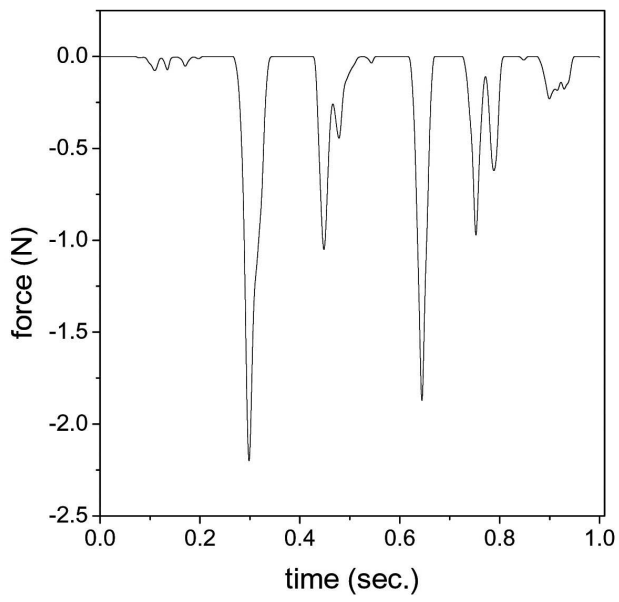

(a) contact force in time domain

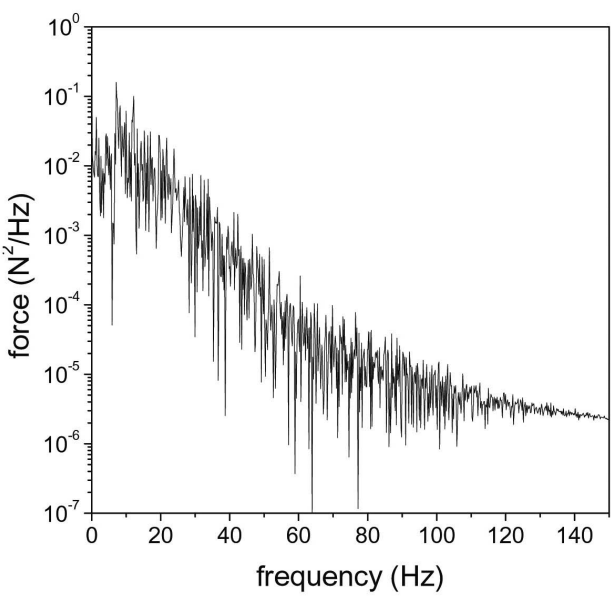

(b) power spectral density of the contact force

Fig. 14. Contact Forces in Time and Frequency Domain

is due to the contact force spectrum shown in Fig. 14, and it is observed that the intensive components are within $50 \mathrm{~Hz}$. The intensity of each contact force frequency component is dependent on the gap size and spring constants. Fig. 15 shows the developed contact force versus motion at the upper support, and this shape is parabolic because the contact force model is defined as such. The displacement of the contacting point with respect to the applied force is also computed, and Fig. 16 shows the root mean square displacement depending on the applied external force. It can be seen that the developed
RMS displacement values are less than $1 \mathrm{~mm}$ and could be reduced when friction is considered.

\section{CONCLUDING REMARKS}

The dynamics of a fuel rod with discretely spaced gap supports have been discussed. For the structure accompanied by a contact phenomenon, the dynamic contact force model needs to be defined. Solutions can be obtained using the Newmark method, which requires the 


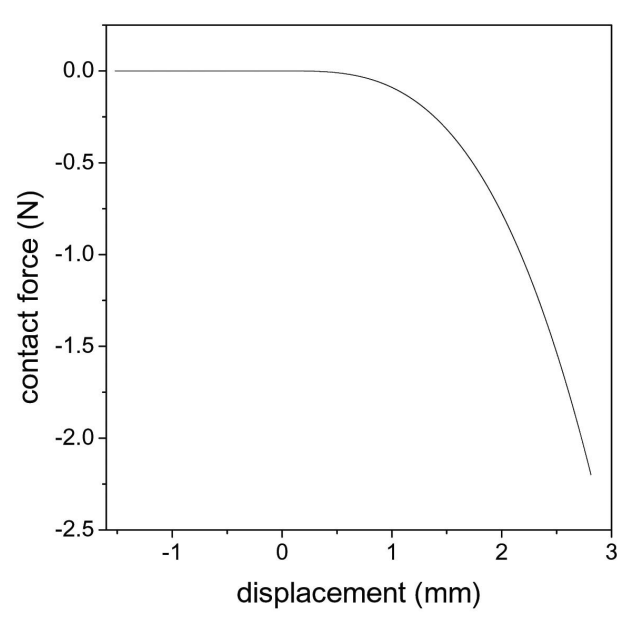

Fig. 15. Force-displacement Curve

contact force a priori. An unknown contact force in the current time can be estimated using the Taylor expansion. However, if the contact force model is not smooth at one point, the contact force at the non-smooth point cannot be predicted. Therefore, a dynamic contact model should be differentiable over the entire displacement field. The model suggested in this work allows differentiation over the displacement field, and using this model, an approximated solution can be found. It has been shown that the suggested model can be derived from the convex and differentiable potential function. Therefore, although it is an approximate solution, a unique solution can be obtained with this model.

Numerical examples have been provided, and it has been shown that the suggested model works well and an abrupt change from a contacting state to a non-contacting state or vice versa can be relieved. For harmonic excitation, the sub-harmonic and super-harmonic components are found in the response signals, resulting from the developed contact force. To simulate a more realistic case, the dynamics of a fuel rod with hardening supports was discussed. The simulation demonstrated that the response is dependent on the contact force as well as an external force. The Rayleigh damping parameter used in this work is an assumed value for a conservative estimation. If reliable information for the damping in a reactor condition is provided, more valuable results are expected.

The fuel rod model can be expanded to cover out-ofplane motion with the friction phenomena, and the work can be helpful in solving such complex structures with contact conditions. During contact, support damping could have an effect because any contacting energy cannot be transmitted entirely. Such support damping is not considered for a simple calculation, but it is expected that a more reliable model can be developed when the impact damping is included. In addition, the sliding force

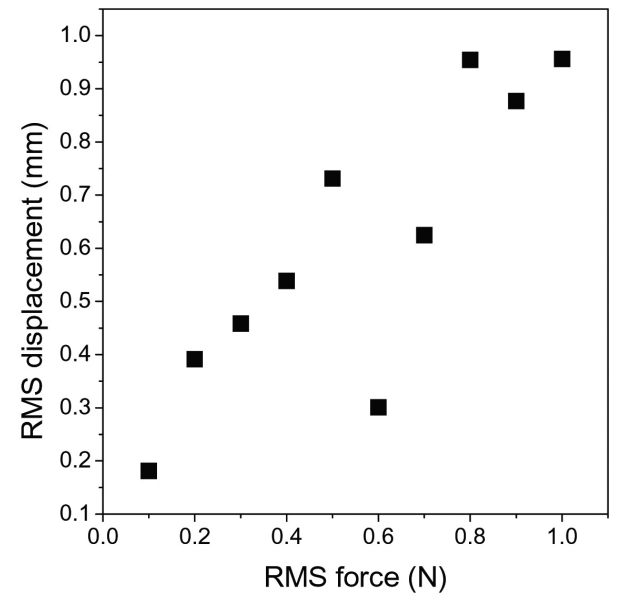

Fig. 16. Distribution of RMS Displacement with Respect to Applied External Forces

that considers friction is required to estimate the wear rates in the fuel rod. The contact force model that considers friction can assist in analyzing fretting wear related problems. Finally, the contact stiffness in the paper is based on the measured static load-deflection characteristics; however, the improved stiffness can be used considering the stress concentration or energy dissipation.

\section{ACKNOWLEDGEMENT}

This work was funded by the Korea Ministry of Knowledge Economy and Korea Ministry of Education, Science and Technology, and the authors express their gratitude.

\section{REFERENCES}

[1] A.J. Molnar, K.M. Vashi, C.W. Gay, "Application of Normal Mode Theory and Pseudoforce Methods to Solve Problems with Nonlinearity", J. Pressure Vessel Tech. 151-156, (1976).

[2 ] S.W. Shaw, P.J. Holmes, "A Periodically Forced Piecewise Linear Oscillator", J. Sound and Vibration 90(1), 129-155, (1983).

[3 ] L. Johansson, "Beam Motion with Unilateral Contact Constraints and Wear of Contact Sites", J. Pressure Vessel Tech. 119, 105-110, (1997).

[4] Xi Tan, R.J. Rogers, "Simulation of Friction in MultiDegree-of-Freedom Vibration Systems", J. Dynamic Systems, Measurement, and Control 120, 144-146, (1998).

[5] N.P. Hoffmann, "Linear Stability of Steady Sliding in Point Contacts with Velocity Dependent and LuGre Type Friction", J. Sound and Vibration 301, 1023-1034, (2006).

[6] M.A. Savi, S. Divenyi, L.F.P. Franca, H.I. Weber, "Numerical and Experimental Investigations of the Nonlinear Dynamics and Chaos in Non-smooth Systems", J. Sound and Vibration 301, 59-73, (2006).

[ 7 ] L. Baillet, S. D'Errico, B. Laulagnet, "Understanding the 
Occurrence of Squealing Noise Using the Temporal Finite Element Method", J. Sound and Vibration 292, 443-460, (2005).

[ 8 ] J. Knudsen, A. R. Massih, "Vibro-Impact Dynamics of a Periodically Forced Beam", J. Pressure Vessel Tech. 122, 210-221, (2000).

[9] Y.B. Gessesse, M.H. Attia, M.O.M. Osman, "On the Mechanics of Crack Initiation and Propagation in ElastoPlastic Materials in Impact Fretting Wear", J. Tribology 126, 393-403, (2004).

[10] F. Axisa, J. Antunes, B. Villard, “Overview of Numerical Methods for Predicting Flow-Induced Vibration”, J. Pressure Vessel Tech. 110, 6-14, (1988).

[11] J.A. Turner, "Non-linear Vibrations of a Beam with Cantilever-Hertzian Contact Boundary Conditions", J. Sound and Vibration 275, 177-191, (2004).

[12] M.A. Hassan, D.S. Weaver, M.A. Dokainish, "The Effects of Support Geometry on the Turbulence Response of Loosely Supported Heat Exchanger Tubes", J. Fluids and Structures 18, 529-554, (2003).

[13] M.A. Hassan, D.S. Weaver, M.A. Dokainish, "A New Tube/Support Impact Model for Heat Exchanger Tubes", J. Fluids and Structures 21, 561-577, (2005).

[14] K. Blakely, W.B. Walton, "Modeling of Nonlinear Elastic Structures Using MSC/NASTRAN", MSC World User's
Conference, (1983).

[15] J. N. Reddy, "An Introduction to Nonlinear Finite element Analysis", Oxford, (2005).

[16] G. Duvaut, J.L. Lions, "Inequalities in Mechanics and Physics", Springer-Velag, (1976).

[17] N. Kikuchi, J.T. Oden, "Contact Problems in Elasticity; A Study of Variational Inequalities and Finite element Methods", SIAM, (1988).

[18] A. Dimarogonas, "Vibration for Engineer", Prentice Hall, (1996).

[19] M.K. Au-Yang, "Flow-Induced Vibration of Power and Process Plant Components", Professional Engineering Publishing, (2001).

[20] C.E. Taylor, M.J. Pettigrew, F. Axisa, B. Villard, "Experimental Determination of Single and Two-phase Cross Flow-Induced Forces on Tube Rows", J. Pressure Vessel Tech. 110, 22-28, (1988).

[21] N.W. Mureithi, S.J. Price, M.P. Paidoussis, "The Post-hopfbifurcation Response of a Loosely Supported Cylinder in an Array Subjected to Cross-flow-part I: Experimental Results", J. Fluids and Structures 8(8), 833-852, (1994).

[22] T.J.R. Hughes, "The Finite Element Method", Dover Publications, (1987).

[23] O. Axelsson, "Finite Element Solution of Boundary Value Problems", Academic Press, (1984). 